\title{
Correlation of Somatostatin Receptor PET/CT Imaging Features and Immunohistochemistry in Neuroendocrine Neoplasms of the Lung: A Retrospective Observational Study
}

Vittoria Rufini ( $\square$ Vittoria.Rufini@unicatt.it)

Universita Cattolica del Sacro Cuore https://orcid.org/0000-0002-1045-0633

Margherita Lorusso

Fondazione Policlinico Universitario Agostino Gemelli IRCCS

Frediano Inzani

Fondazione Policlinico Universitario Agostino Gemelli IRCCS

Tina Pasciuto

Fondazione Policlinico Universitario Agostino Gemelli IRCCS

Elizabeth Katherine Anna Triumbari

Universita Cattolica del Sacro Cuore

Lucia Rosalba Grillo

Ospedale San Camillo: Azienda Ospedaliera San Camillo Forlanini

Filippo Lococo

Universita Cattolica del Sacro Cuore

Stefano Margaritora

Universita Cattolica del Sacro Cuore

Edoardo Pescarmona

Regina Elena Institute: Istituto Regina Elena

Guido Rindi

Universita Cattolica del Sacro Cuore

\section{Research Article}

Keywords: lung NEN, [68Ga]-DOTA-somatostatin analogs, PET/CT, somatostatin receptor subtypes, Ki-67, immunohistochemistry

Posted Date: January 25th, 2022

DOI: https://doi.org/10.21203/rs.3.rs-1273213/v1 
License: (c) (i) This work is licensed under a Creative Commons Attribution 4.0 International License. Read Full License 


\section{Abstract \\ Purpose}

to correlate somatostatin receptor (SSTR) and proliferative activity profile (SSTR2, SSTR5, Ki-67) at immunohistochemistry $(\mathrm{IHC})$ with SSTR-PET/CT imaging features in a retrospective series of lung neuroendocrine neoplasms (NEN). Proliferative activity by Ki-67 and ${ }^{18} \mathrm{~F}$-FDG-PET/CT parameters (when available) were also correlated.

\section{Methods}

Among all consecutive patients who underwent SSTR-PET/CT with ${ }^{68} \mathrm{Ga}$-DOTA-somatostatin analogs (SSA) between July 2011 and March 2020 for lung NEN, 35 patients with a confirmed diagnosis were included. For 16 of them, ${ }^{18} \mathrm{~F}$-FDG-PET/CT studies were available. PET/CT images were reviewed by qualitative and semi-quantitative analyses. Immunohistochemistry for SSTR2, SSTR5, and Ki-67 was assessed. An inferential analysis was performed including kappa statistic and Spearman's rank correlation test.

\section{Results}

Definitive diagnosis consisted of 32 low-intermediate-grade NEN (26 typical carcinoids-G1 and six atypical carcinoids-G2) and 3 high-grade NEN. Positive SSTR2-IHC was found in $63 \%$ of samples while SSTR5-IHC positivity was $17.6 \%$. A correlation between SSTR2-IHC and SSTR-PET/CT was found in 26/35 cases $(74.3 \%, p=0.003)$ : 22 were concordantly positive, 4 concordantly negative. For positive IHC, $100 \%$ concordance with SSTR-PET/CT (both positive) was observed, while for negative IHC concordance (both negative) was $30.8 \%$. In 9 cases, IHC was negative while SSTR-PET/CT was positive, even though

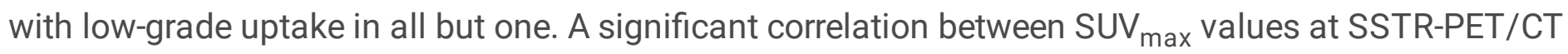
and the SSTR2-IHC scores was found, with low SUV max $_{\text {ax }}$ values corresponding to negative IHC and higher SUV $_{\text {max }}$ values to positive IHC $(p=0.0007)$. When correlating ${ }^{68} \mathrm{Ga}$-DOTA-SSA or ${ }^{18} \mathrm{~F}$-FDG SUV $\max$ values with $\mathrm{Ki}-67$, a significant result was found for ${ }^{18} \mathrm{~F}-\mathrm{FDG}$ only $(p=0.015)$.

\section{Conclusion}

This retrospective study showed an overall good agreement between SSTR2-IHC and tumor uptake at

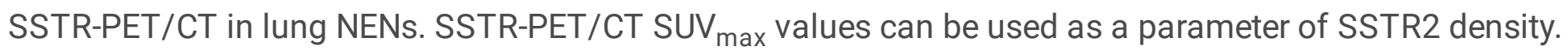
SSTR2-IHC may be proposed as surrogate of SSTR-PET/CT in selected lung NEN patients for clinical decision making when SSTR-PET/CT is not available. 


\section{Introduction}

Neuroendocrine neoplasms (NEN) of the lung represent $20-30 \%$ of all NEN and about $20 \%$ of all lung malignancies [1]. According to the recent World Health Organization (WHO) - International Agency for the Research on Cancer proposal for a standardized NEN nomenclature and the current WHO classification, typical and atypical lung carcinoids ( $T C$ and $A C$ ) belong to the well differentiated neuroendocrine tumor (NET) family and are graded as $G 1$ and $G 2$, respectively while the small cell lung cancer (SCLC) and the large cell neuroendocrine carcinoma (LCNEC) are poorly differentiated neuroendocrine carcinomas (NEC) by default of grade $3[2,3]$. SCLC is by far the largest fraction of lung NEN accounting for $15 \%$ of lung cancer; minor fractions are LCNEC accounting for only $3 \%$ of resected lung cancers as well as TC and AC, which account for $\leq 2 \%$ of all lung malignancies with prevalent TC vs AC [4].

Like NETs of other anatomical sites, most lung NETs express somatostatin receptors (SSTRs), mainly the subtype 2 (SSTR2) [5]. This feature is the basis for the widespread application of diagnostic and therapeutic strategies with "cold" or radiolabeled somatostatin analogues (SSA) in NETs [6, 7]. SSTRpositron emission tomography/computed tomography (SSTR-PET/CT) with ${ }^{68} \mathrm{Ga}$-labelled-SSA is currently used in lung TC-AC/NET for in vivo SSTR characterization and staging $[8,9]$. For the highly proliferative SCLC and LCNEC, ${ }^{18} \mathrm{~F}-\mathrm{FDG}$ PET/CT is the elective functional imaging modality [10]. ${ }^{18} \mathrm{~F}-\mathrm{FDG}$ $\mathrm{PET} / \mathrm{CT}$ has also been explored in lung TC-AC/NET and proved to correlate with cancer cell proliferation $[11,12]$.

In clinical routine, in vitro SSTR expression can be detected on tissue samples by immunohistochemistry (IHC), a reproducible and sensitive routine procedure, which provides information on cellular/subcellular SSTR subtype distribution in tumour cells [13]. An alternative method is reverse transcriptase-polymerase chain reaction (RT-PCR), which demonstrates SSTR mRNA [14]. The correlation of SSTR2-IHC expression vs functional imaging data (in vivo expression) has been assessed in mixed series of patients with various NET types by using SSTR-scintigraphy (Octreoscan $\left.{ }^{\circledR}\right)$ or ${ }^{68} \mathrm{Ga}$-DOTATOC PET/CT $[13,15]$. Limited data are available for lung NEN [5].

Aim of this study was to assess the somatostatin receptor and proliferative activity profile (SSTR2, SSTR5, Ki-67) at IHC in a retrospective selected series of lung NEN, to correlate it with SSTR-PET/CT imaging features, and to specifically assess the potential role of IHC in predicting in vivo SSTR expression. Secondly, we aimed to correlate proliferative activity by Ki-67 with ${ }^{18} \mathrm{~F}$-FDG-PET/CT parameters (when available) in the same cohort.

\section{Materials And Methods}

\section{Study population}

All consecutive patients with a suspected lung NEN who underwent SSTR-PET/CT at our Centre between July 2011 and March 2020, were considered for analysis. Inclusion criteria were: SSTR-PET/CT 
performed for radiological or cytological suspicion of lung NEN; no previous treatment (surgery, chemo- or radiotherapy or "cold" somatostatin analogues); confirmed histopathological diagnosis of lung NEN by lesion biopsy or after surgery; availability of tissue material for pathological review and immunohistochemistry. When available, ${ }^{18} \mathrm{~F}-\mathrm{FDG}$-PET/CT studies performed within a two-month period from SSTR-PET/CT were also reviewed.

All PET/CT studies were performed in the routine clinical practice, for functional characterization of tumor lesions and/or disease staging. This retrospective observational study was approved by the local Ethics Committee (protocol n. 16162/13), and a written informed consent was obtained from all individual participants included in the study.

\section{PET/CT protocols}

PET/CT was performed on a hybrid scanner (Gemini GXL, Philips Medical Systems, Cleveland, Ohio; or Biograph mCT, Siemens Medical Solution, Erlangen, Germany). Low-dose CT scan (120 keV, 80 mA tube current) was acquired for anatomical localization and attenuation correction. For the Siemens Biograph $\mathrm{mCT}, 3 \mathrm{D}$ OSEM reconstruction with PSF modelling/TOF (2 iterations and 21 subsets, voxel size of 3.2 . $3.2 \cdot 5 \mathrm{~mm} 3$ ) was applied. For the Philips Gemini GXL, LOR RAMLA reconstruction (2 iterations and 24 subsets, voxel size: $4 \cdot 4 \cdot 4 \mathrm{~mm}^{3}$ ) was applied.

\section{${ }^{68}$ Ga-DOTA-peptide PET/CT protocol}

Throughout the study period, both ${ }^{68} \mathrm{Ga}$-DOTANOC (from May 2011 to April 2015) and ${ }^{68} \mathrm{Ga-DOTATOC}$ (from May 2015 onwards) were used. ${ }^{68} \mathrm{Ga}$ was obtained from a ${ }^{68} \mathrm{Ge} /{ }^{68} \mathrm{Ga}$ generator (IGG 100; Eckert \& Ziegler Isotope Products, Berlin, Germany), with a nominal activity of $1.85 \mathrm{GBq} .{ }^{68} \mathrm{Ga}$-DOTANOC was produced according to a previously described protocol [16] whereas ${ }^{68} \mathrm{Ga}$-DOTATOC was produced according to the European Pharmacopoeia monograph [17]. PET/CT scans were performed at $45 \pm 10$ minutes after intravenous administration of ${ }^{68} \mathrm{Ga}$-DOTA-peptides ( $2 \mathrm{MBq} / \mathrm{kg}$ ). CT and PET images were acquired from vertex to mid-thigh (4 min/bed position).

\section{${ }^{18}$ F-FDG PET/CT protocol}

${ }^{18} \mathrm{~F}-\mathrm{FDG} \mathrm{PET} / \mathrm{CT}$ was performed after at least 5 hours of fasting. PET images were acquired $60 \pm 10 \mathrm{~min}$ after intravenous injection of ${ }^{18} \mathrm{~F}-\mathrm{FDG}(3.7 \mathrm{MBq} / \mathrm{kg})$. At the time of tracer injection, all patients presented blood glucose levels $<200 \mathrm{mg} / \mathrm{dl}$ and were hydrated with $500 \mathrm{ml}$ of saline solution. CT and PET images were recorded from skull base to mid-thigh ( $3 \mathrm{~min} /$ bed position).

\section{PET/CT imaging interpretation, data collection and analysis}

PET/CT images were independently reviewed by 2 nuclear medicine physicians (M.L. and E.K.A.T.) blinded to clinical reports, who reached a consensus; any disagreements were solved by a third senior reviewer (V.R.). Both qualitative and semi-quantitative analyses were performed. For qualitative analysis, any focal tracer uptake in the lung nodule higher than the surrounding physiologic uptake was considered 
an abnormal (positive) finding. The detection rate (DR) for both tracers was determined by the number of positive studies and the number of patients with lung NEN, according to histological diagnosis. For visual assessment of SSTR-PET/CT images, the Krenning Score, a visual scoring method usually used to assess the degree of tracer uptake on ${ }^{111}$ In-pentetreotide scintigraphy, was also applied [18]. According to this score, abnormal uptake was graded from 0 to 4 by using liver and spleen as reference organs: $0=$ no uptake; 1 = uptake lower than liver; 2 = uptake slightly less than or equal to liver; $3=$ uptake greater than liver; 4 = uptake greater than spleen. Primary tumor size (maximum axial diameter) was measured on the co-registered low dose CT and recorded.

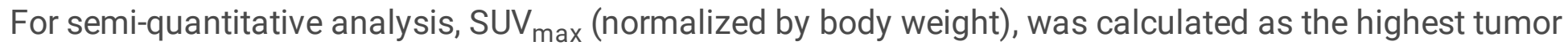
voxel value. To harmonize SUV values obtained by two different PET systems, the EQ•PET referencebased quantification technology was applied [19]. The SUV of the tumor relative to the maximal spleen uptake (for ${ }^{68} \mathrm{Ga}$-DOTA-peptides) or the maximal liver uptake (for ${ }^{18} \mathrm{~F}-\mathrm{FDG}$ ) was calculated by dividing the tumor $S U V_{\text {max }}$ by the spleen $S U V_{\text {max }}\left(S U V_{T / S}\right)$ or liver $S U V_{\max }\left(S U V_{T / L}\right.$, respectively [20]. To limit the influence of the use of two different peptides in semi-quantitative analysis, possible variation in splenic uptake (SUVs) between the two ${ }^{68} \mathrm{Ga}$-peptides was assessed. Furthermore, the ratio between SUV $\max$ of SSTR-PET/CT and SUV max of ${ }^{18}$ F-FDG-PET/CT (SUV max ratio) was calculated [20].

\section{Pathology and immunohistochemistry}

Neoplasms were classified in the four categories of lung NEN according to the WHO 2021 criteria: typical carcinoid TC/NET-G1, atypical carcinoid AC/NET-G2, SCLC and LCNEC [3]. Histopathological information included pathological stage according to the American Joint Committee on Cancer (AJCC) 8th edition Cancer Staging Manual [21], number of mitoses $/ 2 \mathrm{~mm}^{2}$, presence of necrosis and its extension (focal/spotty or extensive) and quantification of intra-tumoral inflammatory component as absent (no lymphocyte detected at x20 magnification), low (<10 lymphocytes detected at x20 magnification field), moderate (10-30 lymphocytes detected at x20 magnification field) and severe (evident lymphocyte aggregates or $>30$ lymphocytes detected at $\times 20$ magnification field).

The following immunohistochemical tests were performed for the study: anti-Ki-67 (MIB1, pre-dilute, Dako, Denmark A/S), Anti-Somatostatin Receptor 2A (SST2) (UMB1, 1:5000, Abcam, USA), AntiSomatostatin Receptor 5 (SST5) (UMB1, 1:5000, Abcam, USA). Ki-67 labelling index was expressed as the percentage of positive cells in highest labelling areas. Immunohistochemical stains for SSTR2 and SSTR5 were assessed as proposed by Volante and coworkers [13] with a semi-quantitative scoring system that considers both the subcellular localization and the extent of the staining, as follows: score 0 , absence of immunoreactivity; score 1, pure cytoplasmic immunoreactivity, either focal or diffuse; score 2, membranous reactivity in $<50 \%$ of tumor cells, irrespective of the presence of cytoplasmic staining; score 3 , circumferential membranous reactivity in $>50 \%$ of tumor cells, irrespective of the presence of cytoplasmic staining. According to Volante and coworkers, score 2 and 3 were considered as positive receptor expression and were grouped together; conversely, score 0 and 1 were considered as negative receptor expression [13]. 


\section{Statistical analysis}

Patient characteristics were described as $\mathrm{n}(\%)$ or median (min-max) as appropriate. The normality of continuous variables was assessed through Shapiro-Wilks test and, in case of normal distribution, mean (with standard deviation) was also provided. Separate descriptive analyses were made according to pathological grading: low-intermediate and high grade. Furthermore, the subset of low-intermediate grade carcinoids was more deeply investigated. Mann-Whitney test and $\chi 2$ or Fisher's exact test, as appropriate, were used to detect differences between TC/NET-G1 and AC/NET-G2 in pathological/IHC and PET findings. Mann-Whitney test was also applied to evaluate the impact of the type of peptide (DOTANOC and DOTATOC) on spleen SUV max $_{\text {ax }}$ evaluation. Kruskall Wallis test was used to assess the association

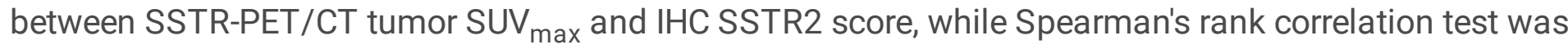
applied to correlate SSTR-PET/CT tumor SUV max $_{\max }$ and proliferation index Ki-67. The agreement between IHC-SSTR2 and PET/CT assessment was evaluated according to kappa statistics [22]. Statistical analysis and graphs were performed using STATA software (STATA/BE 17.0 for Windows, StataCorp LP, College Station, TX 77845, USA). Two-sided tests were applied, and the significance level was set at $p<$ 0.05. No imputation was carried out for missing data.

\section{Results}

Among 551 patients with suspected or confirmed lung NEN who underwent SSTR-PET/CT at our Center between July 2011 and March 2020, 35 patients fulfilled the inclusion criteria. FDG PET/CT was also available for 16 of them. The main clinical, pathological and immunohistochemical features are reported in Table 1. All patients underwent pathological examination by biopsy (11 cases) or surgery ( 24 cases) at a mean of $30.3 \pm 14.7$ days from SSTR-PET/CT. A low-intermediate grade tumor was present in 32 patients (91.4\%), 26 TC/NET-G1 and 6 AC/NET-G2. A high-grade NEN was present in three patients (8.6\%), one LCNEC and two SCLC. 
Table 1

Clinical, pathological and PET/CT characteristics of 35 patients with lung neuroendocrine neoplasia

\section{Characteristics}

Number of patients

Age, years

Median (min-max)

Gender

Male

Female

Pathology

Type of sample

Biopsy

Operative tissue

Histotype

Lung TC/NET G1

Lung AC/NET G2

LCNEC

SCLC

Mitoses $/ 2 \mathrm{~mm}^{2}$, median (min-max)

Necrosis

Absent

Focal/spotty

Extensive

Intra-tumoral inflammatory component
All cases

35

$64(29-82) \quad 62(29-82)$

$65(64-81)$

$14(40.0)$

$21(60.0)$

High grade grade

32 3 


\begin{tabular}{|c|c|c|c|}
\hline Characteristics & All cases & $\begin{array}{l}\text { Low-intermediate } \\
\text { grade }\end{array}$ & High grade \\
\hline 1 & $4(11.4)$ & $4(12.5)$ & $0(0)$ \\
\hline 2 & $11(31.4)$ & $11(34.4)$ & $0(0)$ \\
\hline 3 & $11(31.4)$ & $9(28.1)$ & $2(66.7)$ \\
\hline \multicolumn{4}{|l|}{ SSTR5 } \\
\hline 0 & $\begin{array}{l}27 / 34 \\
(79.4)\end{array}$ & 24/31 (77.4) & $3(100)$ \\
\hline 1 & $1 / 34(2.9)$ & 1/31 (3.2) & $0(0)$ \\
\hline 2 & 6/34 (17.6) & 6/31 (19.4) & $0(0)$ \\
\hline 3 & $0 / 34(0)$ & 0/31 (0) & $0(0)$ \\
\hline \multicolumn{4}{|l|}{ PET/CT findings } \\
\hline $\begin{array}{l}\text { Maximum tumor diameter, mm, median (min- } \\
\max )^{*}\end{array}$ & $\begin{array}{l}19.5(6- \\
100)\end{array}$ & $19.5(6-100)$ & $30(25-52)$ \\
\hline \multicolumn{4}{|l|}{ SSTR-PET/CT } \\
\hline Negative & $4(11.4)$ & $4(12.5)$ & $0(0)$ \\
\hline Positive & $31(88.6)$ & $28(87.5)$ & $3(100)$ \\
\hline Tumor SUV ${ }_{\text {max }}$ median (min-max) & $\begin{array}{l}4.6(0.6- \\
117)\end{array}$ & $4.4(0.6-70.3)$ & $\begin{array}{l}18.3(2.2- \\
117)\end{array}$ \\
\hline \multicolumn{4}{|l|}{${ }^{18} \mathrm{~F}-\mathrm{FDG} \mathrm{PET} / \mathrm{CT}$} \\
\hline Negative & $4 / 16(25.0)$ & 4/14 (28.6) & $0 / 2(0)$ \\
\hline Positive & $\begin{array}{l}12 / 16 \\
(75.0)\end{array}$ & 10/14 (71.4) & $2 / 2(100)$ \\
\hline Tumor SUV max $_{1}$ median (min-max) & $\begin{array}{l}2.7(1.5- \\
17.1)\end{array}$ & $2.5(1.5-10.9)$ & $\begin{array}{l}12.6(8- \\
17.1)\end{array}$ \\
\hline \multicolumn{4}{|c|}{$\begin{array}{l}\text { Results are presented as } \mathrm{n}(\%) \text { except where indicated. For normally distributed variable median (min- } \\
\text { max) was provided. LCNEC: Large Cell Neuroendocrine Carcinoma. SCLC: Small Cell Lung Cancer. } \\
\text { SSTR2: Somatostatin Receptor type } 2 \text {. SSTR5: Somatostatin Receptor type } 5 .{ }^{*} \text { At low-dose CT. SUV: } \\
\text { Standardized Uptake Value. SSTR-PET/CT: Somatostatin Receptor-Positron Emission } \\
\text { Tomography/Computed Tomography. }{ }^{18} \text { F-FDG-PET/CT: }{ }^{18} \text { F-fluorodeoxyglucose-Positron Emission } \\
\text { Tomography/Computed Tomography. }\end{array}$} \\
\hline
\end{tabular}

\section{Pathology}

Data regarding the number of mitoses $/ 2 \mathrm{~mm}^{2}$, presence of necrosis and its extension (focal/spotty or extensive) and presence of intra-tumoral inflammatory component are reported in Table 1. When present 
(44.1\% of samples), the intra-tumoral inflammatory component was low and restricted to lowintermediate grade tumors except for one case of high grade NEN (Table 1).

\section{Immunohistochemistry}

Overall, when considering score 2 and 3 as positive receptor expression, SSTR2 was detected in 22/35 samples (62.9\%), including 2 of 3 NEC. SSTR5 (assessed in 34 samples) was detected in 6 samples (17.6\%), all TC-AC/NET G1-G2. Median Ki-67 was higher in NEC (median value $90 \%$, range $80-90 \%$ ) and lower in NET (median value 4\%, range 1-38\%) (Table 1). When focusing on low-intermediate grade tumors, SSTR2 was detected in 18/26 (69.2\%) TC/NET-G1 (equal distribution of score 2 and 3), and in 2/6 (33.3\%) AC/NET-G2 (all score 2) with no significant difference $(p=0.408)$ (Table 2). SSTR5 was detected in 5/26 (19.2\%) TC/NET-G1 and 1/5 (20\%) AC/NET-G2 (score 2 in all positive samples) with no significant difference $(p=0.968)$. All samples with positive SSTR5 expression were also positive for SSTR2. A significant difference in Ki-67 expression was found between the two histotypes, with higher values of positivity in AC/NET-G2 ( $p=0.0001)$. 
Table 2

Pathological and PET/CT characteristics of 32 low-intermediate grade lung neuroendocrine tumors according to histotypes

\begin{tabular}{|c|c|c|c|c|}
\hline Characteristics & $\begin{array}{l}\text { All cases } \\
n=32\end{array}$ & $\begin{array}{l}\text { TC/ NET G1 } \\
\mathrm{n}=26\end{array}$ & $\begin{array}{l}\text { AC/ NET G2 } \\
n=6\end{array}$ & $\begin{array}{l}p \\
\text { value }\end{array}$ \\
\hline \multicolumn{5}{|l|}{ Pathological and IHC* findings } \\
\hline Intra-tumoral inflammatory component & $14 / 31(45.2)$ & $9 / 26(34.6)$ & $5 / 5(100)$ & 0.007 \\
\hline Ki-67 & $4(1-38)$ & $3(1-10)$ & $21(11-38)$ & 0.0001 \\
\hline SSTR2 & & & & 0.258 \\
\hline 0 & $8(25.0)$ & $5(19.2)$ & $3(50.0)$ & \\
\hline 1 & $4(12.5)$ & $3(11.5)$ & $1(16.7)$ & \\
\hline 2 & $11(34.4)$ & $9(34.6)$ & $2(33.3)$ & \\
\hline 3 & $9(28.1)$ & $9(34.6)$ & $0(0)$ & \\
\hline SSTR5 & & & & 0.905 \\
\hline 0 & $24 / 31(77.4)$ & $\begin{array}{l}20 / 26 \\
(76.9)\end{array}$ & $4 / 5(80.0)$ & \\
\hline 1 & $1 / 31(3.2)$ & $1 / 26(3.8)$ & $0 / 5(0)$ & \\
\hline 2 & 6/31 (19.4) & $5 / 26(19.2)$ & $1 / 5(20.0)$ & \\
\hline 3 & 0/31 (0) & $0 / 26(0)$ & $0 / 5(0)$ & \\
\hline \multicolumn{5}{|l|}{ PET/CT findings } \\
\hline $\begin{array}{l}\text { Maximum tumor diameter, mm, median } \\
(\min -m a x) * \star\end{array}$ & $19(6-100)$ & $17(6-36)$ & $\begin{array}{l}61.5(15- \\
100)\end{array}$ & 0.006 \\
\hline SSTR-PET/CT & & & & 0.461 \\
\hline \multicolumn{5}{|l|}{ Peptide } \\
\hline DOTANOC & $15(46.9)$ & $13(50)$ & $2(33.3)$ & \\
\hline DOTATOC & $17(53.1)$ & $13(50)$ & $4(66.7)$ & \\
\hline Qualitative assessment & & & & 0.732 \\
\hline Negative & $4(12.5)$ & $3(11.5)$ & $1(16.7)$ & \\
\hline Positive & $28(87.5)$ & $23(88.5)$ & $5(83.3)$ & \\
\hline Krenning Score & & & & 0.950 \\
\hline 0 & $4(12.5)$ & $3(11.5)$ & 1 (16.7) & \\
\hline
\end{tabular}




\begin{tabular}{|c|c|c|c|c|}
\hline Characteristics & $\begin{array}{l}\text { All cases } \\
n=32\end{array}$ & $\begin{array}{l}\text { TC/ NET G1 } \\
\mathrm{n}=26\end{array}$ & $\begin{array}{l}\text { AC/ NET G2 } \\
n=6\end{array}$ & $\mathrm{p}_{\text {value }}$ \\
\hline 1 & $12(37.5)$ & $10(38.5)$ & $2(33.3)$ & \\
\hline 2 & $2(6.3)$ & $2(7.7)$ & $0(0)$ & \\
\hline 3 & $10(31.3)$ & $8(30.8)$ & $2(33.3)$ & \\
\hline 4 & $4(12.5)$ & $3(11.5)$ & $1(16.7)$ & \\
\hline Median Tumor SUV $\max$ & $4.4(0.6-70.3)$ & $\begin{array}{l}4.4(0.6- \\
70.3)\end{array}$ & $\begin{array}{l}6.3(1.2- \\
68.5)\end{array}$ & 0.809 \\
\hline Median Spleen SUV $\max$ & $\begin{array}{l}28.3(6.7- \\
44.2)\end{array}$ & $\begin{array}{l}28.8(14.1- \\
44.2)\end{array}$ & $\begin{array}{l}27.7(6.7- \\
41.9)\end{array}$ & 0.901 \\
\hline Median Tumor SUV $V_{\max } /$ Spleen SUV $\max$ & $\begin{array}{l}0.20(0.02- \\
2.76)\end{array}$ & $\begin{array}{l}0.15(0.02- \\
1.96)\end{array}$ & $\begin{array}{l}0.27(0.04- \\
2.76)\end{array}$ & 0.772 \\
\hline \multicolumn{5}{|l|}{${ }^{18} \mathrm{~F}-\mathrm{FDG} \mathrm{PET} / \mathrm{CT}$} \\
\hline Qualitative assessment & & & & 0.505 \\
\hline Negative & $4 / 14(28.6)$ & $4 / 11(36.4)$ & $0 / 3(0)$ & \\
\hline Positive & 10/14 (71.4) & $7 / 11(63.6)$ & $3 / 3(100)$ & \\
\hline Median Tumor SUV $\max$ & $2.5(1.5-10.9)$ & $2.1(1.5-5.8)$ & $\begin{array}{l}7.8(3.3- \\
10.9)\end{array}$ & 0.024 \\
\hline Liver $\mathrm{SUV}_{\max }$ & $3.0(2.0-5.4)$ & $2.9(2.0-5.4)$ & $3.6(3.0-3.9)$ & 0.184 \\
\hline Median Tumor SUV $\max /$ Liver SUV $\max$ & $\begin{array}{l}0.84(0.30- \\
2.78)\end{array}$ & $\begin{array}{l}0.78(0.30- \\
1.88)\end{array}$ & $\begin{array}{l}2.16(1.1- \\
2.78)\end{array}$ & 0.024 \\
\hline $\begin{array}{l}\text { SSTR-PET/CT - }{ }^{18} \text { F-FDG PET/CT SUV } \max \\
\text { ratio }\end{array}$ & $\begin{array}{l}1.35(0.37- \\
20.78)\end{array}$ & $\begin{array}{l}1.73(0.4- \\
20.78)\end{array}$ & $\begin{array}{l}0.37(0.37- \\
0.90)\end{array}$ & 0.024 \\
\hline \multicolumn{5}{|c|}{ 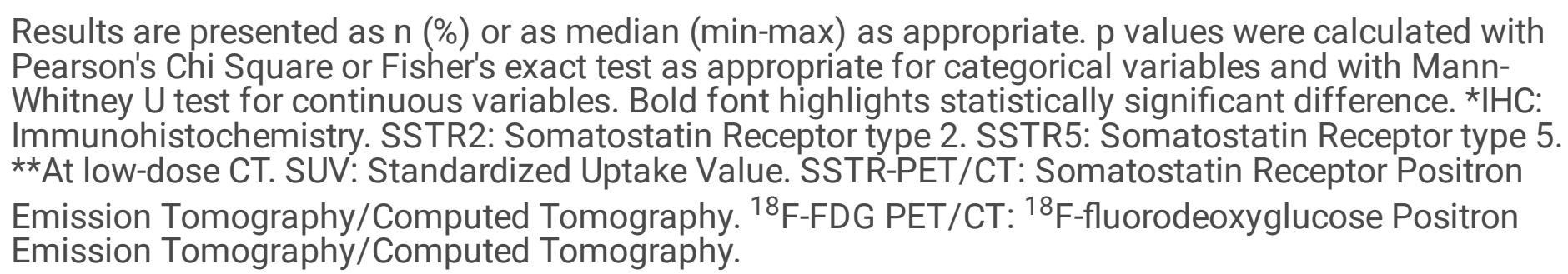 } \\
\hline
\end{tabular}

\section{PET/CT evaluation.}

Qualitative analysis. Considering the overall patient series, SSTR-PET/CT was positive (including Krenning scores $1-4)$ in 31 of 35 cases (88.6\%). The DR for NET was 28/32 (87.5\%); the three NEC were visualized at SSTR-PET/CT, too. Overall, FDG-PET/CT was positive in 12 of the 16 available studies (75.0\%). The DR was 10/14 (71.4\%) for NET; the two NEC studied were also visualized at FDG-PET/CT 
(Table 1). When separately analyzing PET/CT results in the 32 patients with low-intermediate grade tumors, the detection rate of SSTR-PET/CT was 23/26 (88.5\%) for TC/NET-G1 and 5/6 (83.3\%) for AC/NET-G2, with no significant difference. The detection rate of FDG-PET/CT was $7 / 11$ (63.6\%) for TC/NET-G1 and 3/3 (100\%) for AC/NET-G2, with no significant difference. A significant difference in tumor size measured at co-registered low dose CT was found, with larger sizes for AC/NET-G2 $(p=0.006)$ (Table 2).

Semi-quantitative analysis. The analysis of the spleen $\mathrm{SUV}_{\max }$ values showed a median value of 27.3 (range 14.1-41.4) for ${ }^{68} \mathrm{Ga}$ DOTANOC (18 patients) and 29.7 (range 9.0-44.2) for ${ }^{68} \mathrm{Ga-DOTATOC}(17$ patients), with no statistically significant difference between the two peptides. When analyzing SSTR$\mathrm{PET} / \mathrm{CT}$ results in the 32 patients with lung NET, no significant difference was found between TC/NET-G1 and AC/NET-G2 according to PET parameters (tumor $S U V_{\max }$ and $S U V_{T / S}$ ). Higher values of tumor FDG $\mathrm{SUV}_{\max }$ and tumor FDG SUV $/ \mathrm{L}$ were observed in AC/NET G2 compared to TC/NET G1 $(p=0.024)$. Finally, the SUV $V_{\text {max }}$ ratio between ${ }^{68} \mathrm{Ga}$-DOTA-peptides and ${ }^{18} \mathrm{~F}$-FDG PET/CT showed higher values in TC/NET-G1 $(p=0.024)$ (Table 2).

Correlation of PET/CT findings with immunohistochemistry. At qualitative assessment, a concordance between SSTR2 IHC and SSTR-PET/CT was found in 26/35 cases (74.3\%, $\mathrm{p}=0.003)$ : 22 were concordantly positive and 4 were concordantly negative. In detail, for positive IHC (score 2 and 3), $100 \%$ concordance with SSTR-PET/CT (both positive) was observed; for negative IHC (score 0 and 1), 30.8\% concordance (both negative) was observed (Table 3 and Figure 1). In nine cases IHC was negative (four biopsies and five surgical samples) while SSTR-PET/CT was positive with tumor uptake lower than liver uptake in eight of these cases, (4 TC/NET-G1, 3 AC/NET-G2, and 1 LCNEC) and high tracer uptake in a AC/NET-G2 displaying SSTR2 score 1 (Krenning Score $=3$ ). In these nine cases, the median SUV $\mathrm{max}_{\text {was }}$ 2.9 (range 2.0-9.7). No case with positive IHC and negative SSTR-PET/CT was observed. Figure 2A shows the correlation between SSTR2-IHC scores and ${ }^{68} \mathrm{Ga}$-DOTA-peptide SUV $\mathrm{max}_{\max }$ with a significant difference among the SUV $V_{\max }$ values for different IHC scores $(p=0.0007)$. Figure $2 B$ shows the correlation between

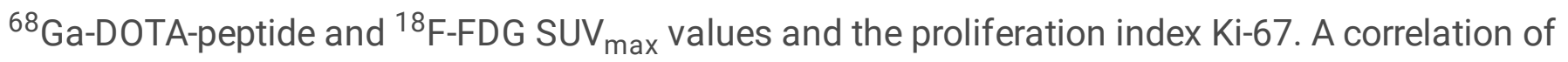
moderate statistical significance between $\mathrm{SUV}_{\max }$ and Ki-67 was found for ${ }^{18} \mathrm{~F}-\mathrm{FDG}$ only (rho $=0.593$; $p=0.015)$. When separately analyzing well-differentiated/low grade tumors, a correlation of strong statistical significance between SUV max $_{\text {ax }}$ and $\mathrm{Ki}-67$ was again found for ${ }^{18} \mathrm{~F}-\mathrm{FDG}$ only in lung AC/NET-G2 $($ rho $=1 ; p<0.0001)$, even though the patients observed were only three (Figure 3 ). 
Table 3

Concordance between SSTR-PET/CT qualitative assessment and SSTR2 immunohistochemistry

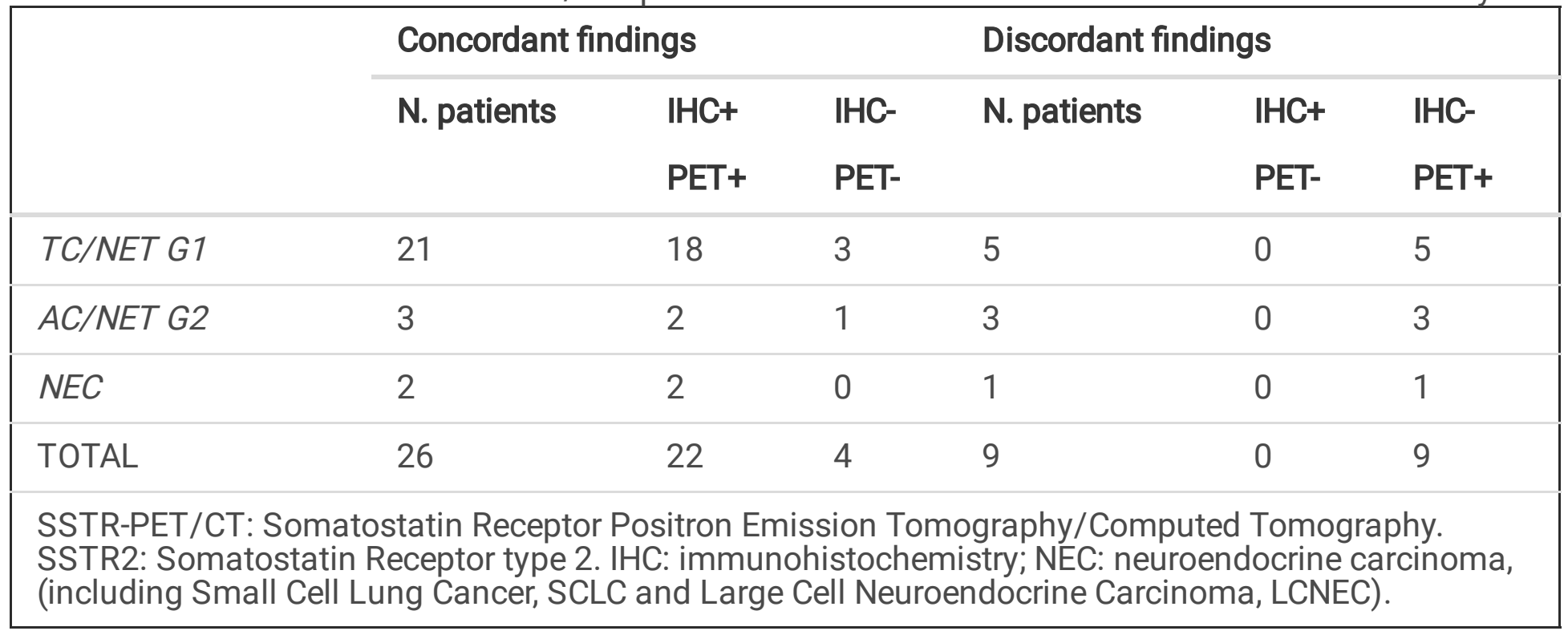

\section{Discussion}

In this study we found an overall good agreement $(74.3 \%, \mathrm{p}=0.003)$ between in vitro SSTR expression and tumor uptake of ${ }^{68} \mathrm{Ga}$-DOTA-SSA at qualitative assessment in a retrospective series of patients with lung NEN. For positive SSTR expression, $100 \%$ concordance was found. Similarly, higher SUV max $_{\text {values }}$ corresponded to membranous staining, which indicates positive receptor expression [13]. These findings may impact on clinical practice in cases where SSTR-PET/CT is not available for preoperative and staging purposes. In these cases, the demonstration of membranous staining in NET biopsy may surrogate SSTR-PET/CT for treatment with "cold" or radiolabeled SSA [14, 23-25]. Indeed, ${ }^{68}$ Ga-DOTASSA PET/CT, showing high affinity for SSTR2 (higher binding affinity of the analog DOTATATE followed by DOTANOC and then by DOTATOC) and, with some ligands, also for SSTR3 (DOTANOC) and/or SSTR5 (DOTANOC and DOTATOC), is considered the most reliable method for in vivo assessment of somatostatin receptor status [26]. It provides information on the presence of SSTR and its affinity for the radio-ligand and allows tumor localization with high sensitivity. Moreover, the intensity of tracer uptake is used to tailor treatment with SSA and select patients suitable for peptide receptor radionuclide therapy (PRRT) [23-25].

Most of the patients included in the present cohort (91\%) had a well differentiated lung NET, a finding that is a consequence of the retrospective nature of our study as well as the inclusion criteria. Indeed, patients with poorly differentiated/high grade lung NEN are rarely submitted to PET/CT studies for staging purposes. Among lung NET patients, we found an $81 \%$ prevalence of TC/NET-G1, which is in line with literature data [4].

For in vitro studies, we applied the scoring system proposed by Volante and coworkers [13] which evaluates the subcellular localization (membranous or cytoplasmatic) and the extent of staining. In line with previous data, SSTR2 was the predominantly expressed receptor subtype with positive findings in 
$63 \%$ of samples [5]. SSTR5 was poorly represented with a positive rate of $17.6 \%$, not improving the overall concordance between in vitro and in vivo results. Based on this, further considerations on our findings are essentially based on SSTR2 expression. Scores 2-3 were predominantly detected in TC/NETG1 ( $62 \%$ vs $33 \%$ of AC/NET-G2), even though the difference was not statistically significant, likely due to the few samples of AC/NET-G2 analyzed. In this histotype, score 3 indicating the highest receptor density was never observed in the present series. Two of three NEC samples expressed SSTR2, score 3 in both cases, a finding observed in up to $25 \%$ of NEC at various anatomical sites [27].

The detection rate of SSTR-PET/CT was $88.5 \%$ for TC/NET-G1 and $83.3 \%$ for AC/NET-G2, with no significant difference. Therefore, a little amount of SSTR-negative tumors belonged to both histotypes and, in other words, SSTR in vivo expression was independent from tumor grade in well-differentiated lung NEN. Even when analyzing semi-quantitative data, no significant difference was found between TC/NET-G1 and AC/NET-G2 according to SSTR-PET parameters (tumor SUV $\mathrm{max}_{\text {max }}$ and SUV $\mathrm{V}_{\mathrm{T} / \mathrm{S}}$ ). These observations are in line to what found in corresponding tissue samples at IHC.

We didn't find any case of positive IHC and negative SSTR-PET/CT, thus confirming the role of reference method assigned to functional imaging with radio-receptor PET/CT. Conversely Papotti et al. and Volante et al. reported cases of positive $\mathrm{IHC}$ and negative radio-receptor scintigraphy, a technique that is characterized by lower spatial resolution and lower sensitivity than PET/CT imaging $[5,13]$. Moreover, the binding affinity for SSTR2 and SSTR5 of the radiolabeled SSA is significantly affected by structural changes of the octapeptide as well as the choice of chelator (DTPA vs DOTA) or metal used for labeling, with a clear advantage for ${ }^{68} \mathrm{Ga}$-DOTA-labeled compounds over ${ }^{111} \mathrm{In}$-DTPA-octreotide in terms of binding affinity and consequently of imaging findings [28].

As for the IHC negative cases of the present series, only $31 \%$ concordance was found since SSTR-PET/CT was positive in nine cases, even though with low-grade uptake (Krenning Score $=1$ ) in all but one of them. Similar discrepancies have been previously observed, even though in mixed series of NEN patients studied by PET/CT [15] or in patients with lung NEN submitted to ${ }^{111}$ In-DTPA-octreotide scintigraphy [5, 13]. In our series, the visualization (although with low uptake) of a lung lesion with negative SSTR2-IHC, could be partly explained by the binding affinity of ${ }^{68} \mathrm{Ga}$-DOTATOC and ${ }^{68} \mathrm{Ga}$-DOTANOC to SSTR2, higher than that of Octreoscan ${ }^{\circledR}[28]$. In any case, current imaging selection criteria do not consider PRRT for lesions showing no or low uptake, so most negative cases for SSTR at IHC should remain unsuitable (eight out of nine in this series) for PRRT despite slightly in vivo positivity.

In addition to neuroendocrine tumor cells, other non-neoplastic cells such as lymphoid and endothelial cells express SSTRs $[29,30]$. As functional radio-receptor imaging cannot define the cellular receptor localization, we quantified the intra-tumoral inflammatory component on tissue samples as potential source of positive SSTR-PET/CT imaging. When present ( $44.1 \%$ of samples), the intra-tumoral inflammatory component was low, making it unlikely to be the determinant of radioligand uptake. Conversely, the discordant finding of SSTRs expression in vivo vs in vitro, could be explained by SSTRs 
expression levels below the detection limit of IHC. On this line, the low values of SUV $\mathrm{max}_{\text {ax }}$ observed in discrepant cases would support this potential explanation.

A relevant observation of our study was that in vitro SSTR scores and SUV values at SSTR-PET/CT proceeded at the same rate. Cytoplasmatic staining, indicating negative receptor expression, matched low SUV values, and membranous staining, indicating positive receptor expression, matched higher SUV values $(p=0.0007)[13]$. Therefore, SUV values can be used as a parameter of SSTR2 density. Miederer and coworkers in a mixed series of NEN [15] and Kaemmerer and coworkers in gastro-entero-pancreatic NET reported a similar highly significant correlation [31]. However, some discrepant results were also observed in our series, as one of 13 tumors with IHC score 0-1 showed high SUV values (9.7 in one AC/NET-G2) and 3 of 22 tumors with IHC score 2-3 showed low SUV values ( $<4.0$, all TC/NET-G1). These discrepancies, on the tissue side may reflect non-standard tissue handling for histology processing with resulting poor SSTRs preservation, and on the in vivo side, undetermined reduced SSTRs expression status or SSTRs blockade.

Besides analyzing the receptor profile, we also studied proliferative activity by Ki-67 staining, which, as expected, proved to be an important discriminant factor between TC/NET-G1 and AC/NET-G2, with higher values of positivity in AC/NET-G2 ( $p=0.0001)$. Parallel to this observation, a correlation of strong

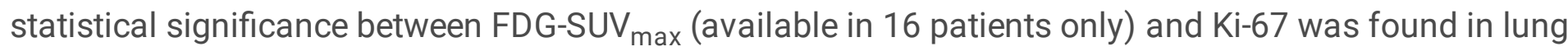
AC/NET-G2 (rho $=1 ; p<0.0001$ ), even though the calculation could be affected by the small number of patients studied with ${ }^{18}$ F-FDG-PET/CT. Conversely, we did not find any correlation between SUV $\mathrm{max}_{\text {max }}$ at SSTR-PET/CT and Ki-67 values, both in the overall series and when analyzing separately TC/NET-G1 and AC/NET-G2. These findings are in agreement with those reported by Campana and coworkers in a mixed series of NET patients studied with ${ }^{68} \mathrm{Ga}$-DOTANOC [32].

In line with our previous study, the use of $\mathrm{SUV}_{\max }$ ratio between ${ }^{68} \mathrm{Ga}$-DOTA-peptides and ${ }^{18} \mathrm{~F}$-FDG PET/CT allowed the distinction between TC/NET-G1 and AC/NET-G2 in those lung lesions that are visualized by both tracers. This could be a valid help in surgical management, potentially influencing the extension of parenchymal resection and lymph node dissection, considering that the differentiation between the two histotypes is rarely feasible through pre-operative biopsies [20].

This study has some limitations. First, its retrospective nature. Second, two different SSA were used as radio-ligand, with different affinities to SSTR. To assess the influence on pharmacokinetics of the two different peptides, we calculated possible variations in splenic uptake; no significant differences were observed. Third, tissue handling though following rigid standard procedures may slightly vary between cases, with improper receptor antigen preservation and poor resulting IHC. Nonetheless, this study was performed in a highly selected and well annotated series of lung NEN, while available literature data are relatively old and not specifically targeting lung $\operatorname{NEN}[5,13,15,31]$.

In conclusion, information on comparative SSTRs expression in vitro and in vivo in patients with lung NEN is limited. This retrospective study showed an overall good agreement between SSTR2-IHC on tissue 
and tumor uptake at ${ }^{68} \mathrm{Ga}$-SSA-PET/CT in patients with lung NEN. PET/CT with ${ }^{68} \mathrm{Ga}$-SSA confirmed to be the most reliable method for in vivo assessment of receptor status. In clinical practice, SSTR-PET/CT SUV $_{\max }$ values can be used as a parameter of SSTR2 density. Membranous staining in tissue samples is feasible and informative for follow-up studies with SSTR-PET/CT and potentially for treatment with "cold" or radiolabeled SSA analogs. SSTR2-IHC could be proposed as surrogate of SSTR-PET/CT in selected lung NEN patients for effective clinical decision making when SSTR-PET/CT is not available.

\section{Declarations}

\section{Funding}

This work was supported by internal university grant (Università Cattolica Line D.1 2013-70201140) to Vittoria Rufini and internal university grant (Università Cattolica Line D.1 2018-R4124500740) to Guido Rindi

Margherita Lorusso, Frediano Inzani, Tina Pasciuto, Elizabeth KA Triumbari, Lucia Rosalba Grillo, Filippo Lococo, Stefano Margaritora, and Edoardo Pescarmona declare that no funds, grants, or other support were received during the preparation of this manuscript.

\section{Competing interest}

Financial interests: Filippo Lococo and Stefano Margaritora received speaker and consultant honoraria from Astrazeneca. Guido Rindi received speaker and consultant honoraria from Company AAA and Bracco.

Vittoria Rufini, Margherita Lorusso, Frediano Inzani, Tina Pasciuto, Elizabeth KA Triumbari, Lucia Rosalba Grillo, and Edoardo Pescarmona declare no financial interests.

\section{Author contributions}

All authors contributed to the study conception and design. Material preparation and data collection were performed by Margherita Lorusso, Frediano Inzani, Filippo Lococo, Lucia Rosalba Grillo, Edoardo Pescarmona and Elizabeth K.A. Triumbari. Data analysis was performed by Tina Pasciuto. The first draft of the manuscript was written by Vittoria Rufini, Margherita Lorusso, Guido Rindi, and Frediano Inzani. All authors commented on previous versions of the manuscript. All authors read and approved the final manuscript.

\section{Data availability}

The datasets generated during and/or analysed during the current study are available from the corresponding author on reasonable request.

\section{Ethics approval}


This study was performed in line with the principles of the Declaration of Helsinki. Approval was granted by the Ethics Committee of Fondazione Policlinico Universitario Agostino Gemelli IRCCS - Università Cattolica del Sacro Cuore (July 18, 2013 - No. 16162/13)

\section{Consent to participation}

Informed consent was obtained from all individual participants included in the study.

\section{References}

1. G. Fink, T. Krelbaum, A. Yellin, Bendayan D, Saute M, Glazer M, et al. Pulmonary carcinoid: presentation, diagnosis, and outcome in 142 cases in Israel and review of 640 cases from the literature, Chest 2001; 119:1647-51. https://doi.org/10.1378/chest.119.6.1647.

2. Rindi G, Klimstra DS, Abedi-Ardekani B, Asa SL, Bosman FT, Brambilla E, et al. A common classification framework for neuroendocrine neoplasms: an International Agency for Research on Cancer (IARC) and World Health Organization (WHO) expert consensus proposal. Mod Pathol. 2018; 31:1770-86. https://doi.org/10.1038/s41379-018-0110-y.

3. Travis WD, Beasley MB, Cree IA, Papotti M, Rekhtman N, Rossi G. Lung neuroendocrine neoplasms: Introduction. In: WHO Classification of Tumours Series. Thoracic Tumours, 5th Edition ed. Lyon: IARC Press; 2021. pp. 127-129.

4. Papotti M, Brambilla E, Dingemans AC, Fernandez-Cuesta L, Lantuejoul S, MacMahon $\mathrm{H}$, et al. Carcinoid/Neuroendocrine tumour of the lung. In: WHO Classification of Tumours Series. Thoracic Tumours, 5th Edition ed. Lyon: IARC Press; 2021. pp. 133-138.

5. Papotti M, Croce S, Bellò M, Bongiovanni M, Allìa E, Schindler M, et al. Expression of somatostatin receptor types 2, 3 and 5 in biopsies and surgical specimens of human lung tumours. Correlation with preoperative octreotide scintigraphy. Virchows Arch. 2001; 439:787-

97. https://doi.org/10.1007/s004280100494.

6. Smit Duijzentkunst DA, Kwekkeboom DJ, Bodei L. Somatostatin receptor 2-targeting compounds. J Nucl Med. 2017;58(Suppl 2):54S-60S. https://doi.org/10.2967/jnumed.117.191015.

7. Ambrosini V, Kunikowska J, Baudin E, Bodei L, Bouvier C, Capdevila J, et al. Consensus on molecular imaging and theranostics in neuroendocrine neoplasms. Eur J Cancer. 2021; 146:5673. https://doi.org/10.1016/j.ejca.2021.01.008.

8. Singh S, Bergsland EK, Card CM, Hope TA, Kunz PL, Laidley DT, et al. Commonwealth neuroendocrine tumour research collaboration and the North American neuroendocrine tumor society guidelines for the diagnosis and management of patients with lung neuroendocrine tumors: An international collaborative endorsement and update of the 2015 European Neuroendocrine Tumor Society Expert Consensus Guidelines. J Thorac Oncol. 2020; 15:1577-

98. https://doi.org/10.1016/j.tho.2020.06.021. 
9. Jiang Y, Hou G, Cheng W. The utility of ${ }^{18} \mathrm{~F}-\mathrm{FDG}$ and ${ }^{68} \mathrm{Ga}$-DOTA-Peptide PET/CT in the evaluation of primary pulmonary carcinoid: A systematic review and meta-analysis. Medicine (Baltimore). 2019; 98:e14769. https://doi.org/10.1097/MD.0000000000014769.

10. Martucci F, Pascale M, Valli MC, Pesce GA, Froesch P, Giovanella L, et al. Impact of ${ }^{18}$ F-FDG PET/CT in staging patients with small cell lung cancer: A systematic review and meta-analysis. Front Med (Lausanne). 2020; 6:336. https://doi.org/10.3389(fmed.2019.00336.

11. Kaira K, Murakami H, Endo M, Ohde Y, Naito T, Kondo H, et al. Biological correlation of ${ }^{18} \mathrm{~F}$-FDG uptake on PET in pulmonary neuroendocrine tumors. Anticancer Res. 2013; 33:4219-28

12. Lococo F, Cesario A, Paci M, Filice A, Versari A, Rapicetta C, et al. PET/CT assessment of neuroendocrine tumors of the lung with special emphasis on bronchial carcinoids. Tumour Biol. 2014; 35:8369-77. https://doi.org/10.1007/s13277-014-2102-y.

13. Volante M, Brizzi MP, Faggiano A, La Rosa S, Rapa I, Ferrero A, et al. Somatostatin receptor type 2A immunohistochemistry in neuroendocrine tumors: a proposal of scoring system correlated with somatostatin receptor scintigraphy. Mod Pathol. 2007; 20:1172-

82. https://doi.org/:10.1038/modpathol.3800954.

14. Volante M, Bozzalla-Cassione F, Papotti M. Somatostatin receptors and their interest in diagnostic pathology. Endocr Pathol. 2004; 15:275-91. https://doi.org/10.1385/ep:15:4:275

15. Miederer M, Seidl S, Buck A, Scheidhauer K, Wester HJ, Schwaiger M, Perren A. Correlation of immunohistopathological expression of somatostatin receptor 2 with standardized uptake values in ${ }^{68} \mathrm{Ga}$-DOTATOC PET/CT. Eur J Nucl Med Mol Imaging. 2009; 36:48-

52. https://doi.org/10.1007/s00259-008-0944-5.

16. Treglia G, Castaldi P, Villani MF, Perotti G, de Waure C, Filice A et al. Comparison of ${ }^{18} \mathrm{~F}-\mathrm{DOPA},{ }^{18} \mathrm{~F}$ FDG and ${ }^{68} \mathrm{Ga}$-somatostatin analogue PET/CT in patients with recurrent medullary thyroid carcinoma. Eur J Nucl Med Mol Imaging. 2012; 39:569-80. https://doi.org/10.1007/s00259-0112031-6.

17. European Pharmacopeia 7.7 (01/2013:2482 Gallium (68Ga) Edotreotide injection) 2011; 23: 310.

18. Hofman MS, Lau WF, Hicks RJ. Somatostatin receptor imaging with ${ }^{68} \mathrm{Ga}$ DOTATATE PET/CT: clinical utility, normal patterns, pearls, and pitfalls in interpretation. Radiographics. 2015; 35:50016. https://doi.org/10.1148/rg.352140164.

19. Quak E, le Roux PY, Hofman MS, Robin P, Bourhis D, Callahan J, et al. Harmonizing FDG PET quantification while maintaining optimal lesion detection: prospective multicentre validation in 517 oncology patients. Eur J Nucl Med Mol Imaging. 2015; 42:2072-82. https://doi.org/10.1007/s00259015-3128-0.

20. Lococo F, Perotti G, Cardillo G, De Waure c, Filice A, Graziano P, et al. Multicenter comparison of ${ }^{18} \mathrm{~F}$ FDG and ${ }^{68} \mathrm{Ga}$-DOTA-peptide PET/CT for pulmonary carcinoid. Clin Nucl Med. 2015; 40:e1839. https://doi.org/10.1097/RLU.0000000000000641.

21. Amin M.B. AJCC Cancer Staging Manual, VIII ed.; Springer-Verlag: Berlin/Heidelberg, Germany, 2017. 
22. Landis, J. R., and G. G. Koch. 1977a. The measurement of observer agreement for categorical data. Biometrics 33: 159-174. http://doi.org/10.2307/2529310.

23. Lenotti E, Alberti A, Spada F, Amoroso V, Maisoneuve P, Grisanti S, et al. Outcome of patients with metastatic lung neuroendocrine tumors submitted to first line monotherapy with somatostatin analogs. Front Endocrinol (Lausanne). 2021;

12:669484. https://doi.org/10.3389/fendo.2021.669484

24. Mirvis E, Toumpanakis C, Mandair D, Gnanasegaran G, Caplin M, Navalkissoor S. Efficacy and tolerability of peptide receptor radionuclide therapy (PRRT) in advanced metastatic bronchial neuroendocrine tumours (NETs). Lung Cancer. 2020; 150:70-

75. https://doi.org/10.1016/j.lungcan.2020.10.005.

25. Park S, Parihar AS, Bodei L, Hope TA, Mallak N, Millo C, et al. Somatostatin Receptor Imaging and Theranostics: Current Practice and Future Prospects. J Nucl Med. 2021: 62:1323-1329. https://doi.org/10.2967/jnumed.120.251512.

26. Sundin A, Arnold R, Baudin E, Cwikla JB, Eriksson B, Fanti S, et al. ENETS Consensus Guidelines for the standards of care in neuroendocrine tumors: radiological, nuclear medicine \& hybrid imaging. Neuroendocrinology 2017; 105: 212-244. https://doi.org/10.1159/000471879.

27. Konukiewitz B, Schlitter AM, Jesinghaus M, Pfister D, Steiger K, Segler A, et al. Somatostatin receptor expression related to TP53 and RB1 alterations in pancreatic and extrapancreatic neuroendocrine neoplasms with a Ki67-index above 20. Mod Pathol. 2017; 30:587598. https://doi.org/10.1038/modpathol.2016.21724.

28. Reubi JC, Schär JC, Waser B, Wenger S, Heppeler A, Schmitt JS, et al. Affinity profiles for human somatostatin receptor subtypes SST1-SST5 of somatostatin radiotracers selected for scintigraphic and radiotherapeutic use. Eur J Nucl Med. 2000; 27:273-

82. https://doi.org/10.1007/s002590050034.

29. Lichtenauer-Kaligis EG, Dalm VA, Oomen SP, Mooij DM, van Hagen PM, Lamberts SW, et al. Differential expression of somatostatin receptor subtypes in human peripheral blood mononuclear cell subsets. Eur J Endocrinol. 2004; 150:565-77. https://doi.org/10.1530/eje.0.1500565. Erratum in: Eur J Endocrinol. 2005; 152:325.

30. Reubi JC, Mazzucchelli L, Laissue JA. Intestinal vessels express a high density of somatostatin receptors in human inflammatory bowel disease. Gastroenterology. 1994; 106:9519. https://doi.org/10.1016/0016-5085(94)90754-4.

31. Kaemmerer D, Peter L, Lupp A, Schulz S, Sänger J, Prasad V, et al. Molecular imaging with $\triangle \mathbb{G}$ Ga-SSTR $\mathrm{PET} / \mathrm{CT}$ and correlation to immunohistochemistry of somatostatin receptors in neuroendocrine tumours. Eur J Nucl Med Mol Imaging. 2011;38:1659-68. https://doi.org/10.1007/s00259-011-18465.

32. Campana D, Ambrosini V, Pezzilli R, Fanti S, Labate AM, Santini D, et al. Standardized uptake values of ${ }^{68} \mathrm{Ga}$-DOTANOC PET: a promising prognostic tool in neuroendocrine tumors. J Nucl Med. 2010; 51:353-9. https://doi.org/10.2967/jnumed.109.066662. 


\section{Figures}

A

IHC negative $(n=13)$
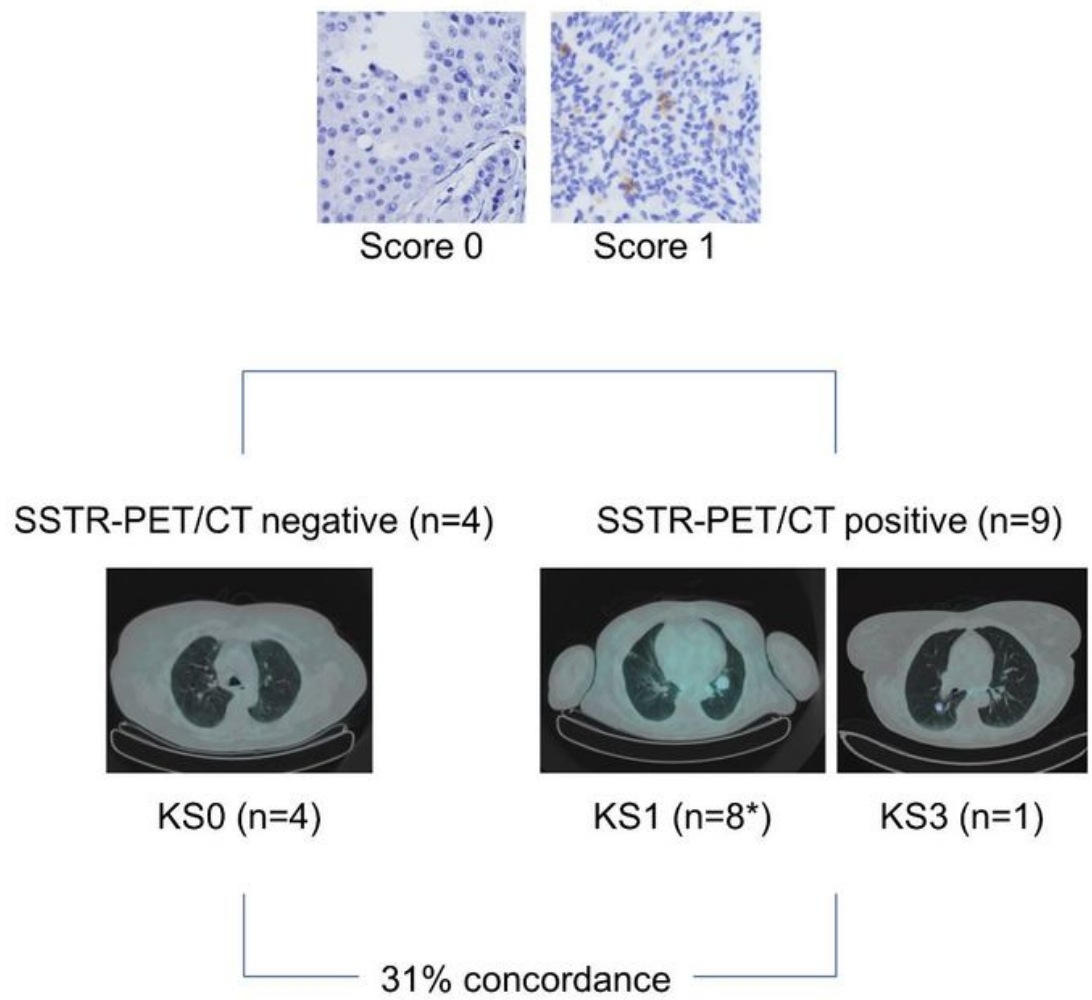

B

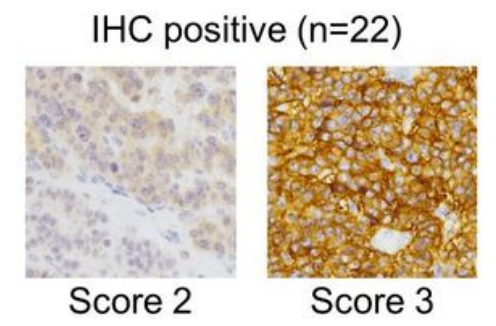

${ }^{68} \mathrm{Ga}$ PET/CT positive $(n=22)$

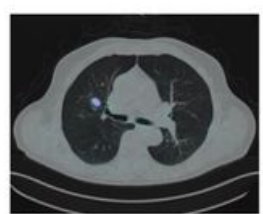

KS1 $(n=5)$

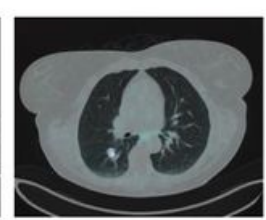

$\mathrm{KS} 2(\mathrm{n}=2)$

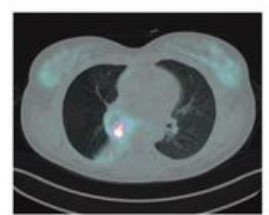

KS3 (n=10§)

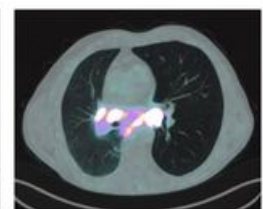

KS4 ( $n=5 \S)$

$100 \%$ concordance

\section{Figure 1}

Concordant and discordant findings of pathological and functional assessments with representative images of SSTR2-IHC and SSTR-PET/CT (fused transaxial images) corresponding to the various IHC scores and Krenning scores. A) Patients with negative SSTR2-IHC (score 0 and 1) and B) Patients with positive SSTR2-IHC (score 2 and 3). *One case of large cell neuroendocrine carcinoma; §one case of small cell lung cancer. 

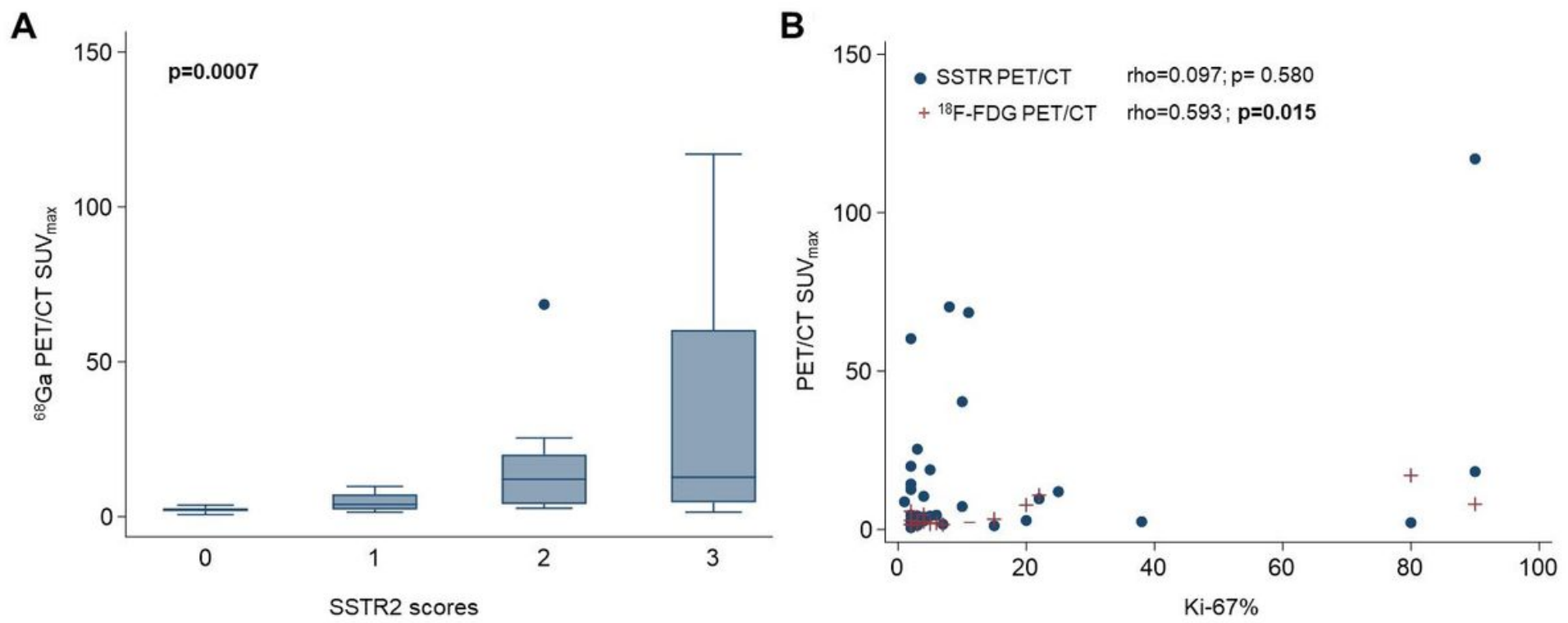

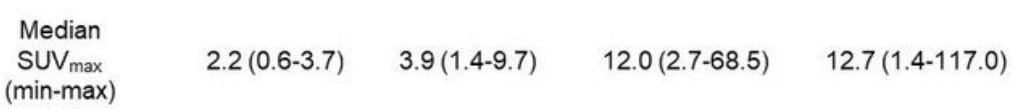

\section{Figure 2}

A) Box plot of SSTR-PET/CT SUV $\max$ by SSTR-2 IHC scores ranging from 0 to 3 . The boxes indicate medians with upper (Q3) and lower quartiles (Q1); the upper and lowers bars define values between Q3 Q3+3/2(Q3-Q1) and Q1-3/2(Q3-Q1) - Q1, respectively; dots indicate outliers. P value was evaluated with

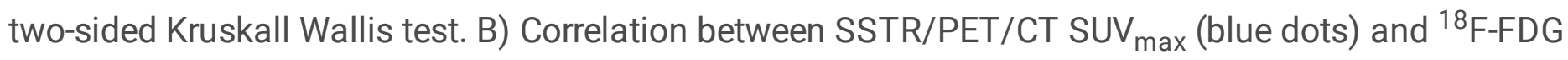

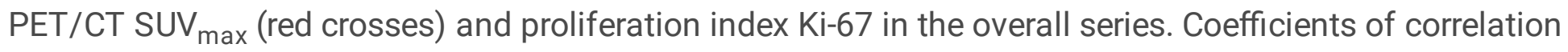
(rho) and $p$ values were calculated with two-sided Spearman's rank correlation test. 


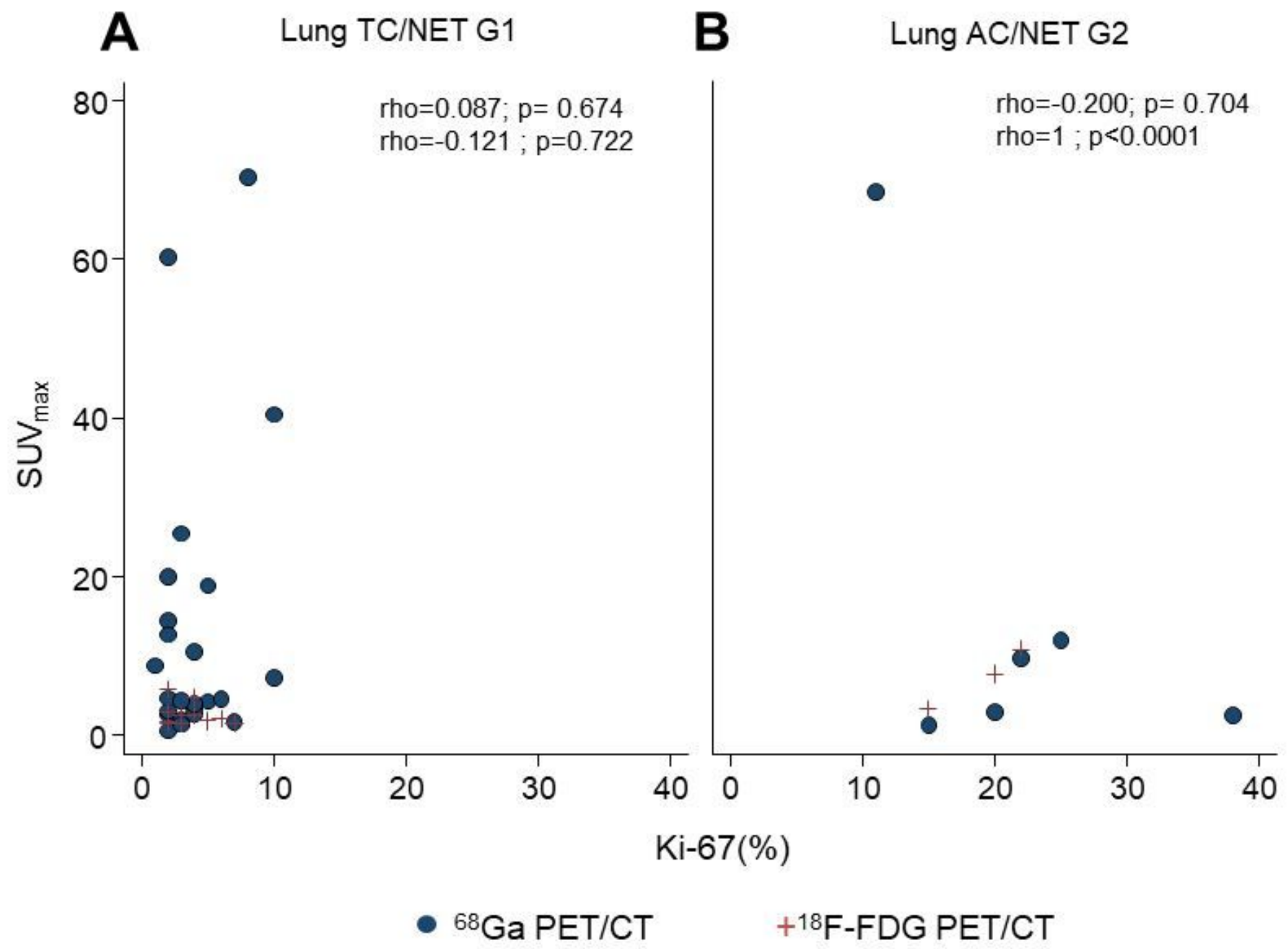

Figure 3

Correlation between SSTR/PET/CT SUV max (blue dots) and ${ }^{18}$ F-FDG PET/CT SUV max $_{\text {(red crosses) and }}$ proliferation index Ki-67 in typical carcinoids (TC/NET-G1) (A) and atypical carcinoids (AC/NET-G2) (B). Coefficients of correlation (rho) and $p$ values were calculated with two-sided Spearman's rank correlation test. 